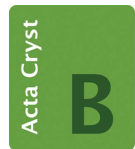

STRUCTURAL SCIENCE CRYSTAL ENGINEERING MATERIALS

ISSN 2052-5206

Received 21 May 2015

Accepted 21 May 2015

Keywords: aperiodic crystals; incommensurate modulated structures.

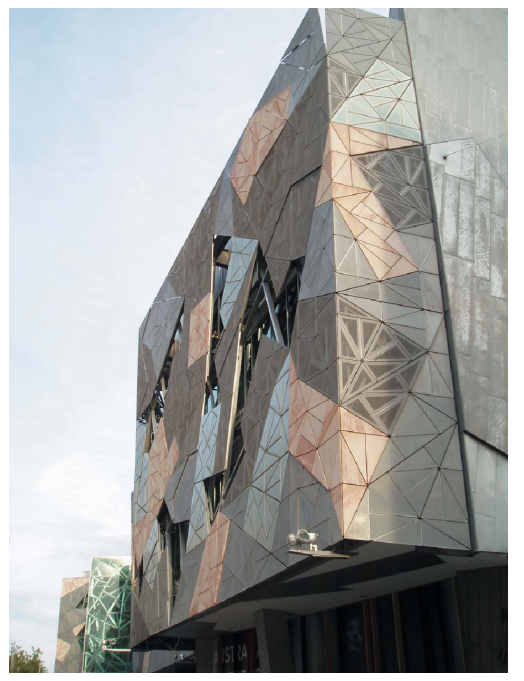

C 2015 International Union of Crystallography

\section{Structures beyond superspace}

\author{
Marjorie Senechal*
}

Burton 211, Smith College, Northampton, MA 01063, USA. *Correspondence e-mail: senechal@smith.edu

After Shechtman et al. (1984) published the paradigm-shattering 'Metallic Phase with Long-Range Orientational Order and No Translational Symmetry', it was evident (to all but quasicrystal-deniers) that the textbook answer to 'what is a crystal?' no longer sufficed. In response, the IUCr charged its newly appointed Commission on Aperiodic Crystals with finding a sufficiently broad definition of 'crystal', one that went beyond three-dimensional periodicity to include quasicrystals and other unexpected structures that might be discovered. No quasicrystal structure had yet been solved, but scientists had already broadened the crystal structure kingdom: de Wolff (1974) and Janner \& Janssen (1977) had already shown that modulated crystals, with incommensurate periodicities, could be rationalized in a four-dimensional 'superspace'. Accordingly, several Commission members argued that 'crystal' should mean a solid with Bragg peaks and a superspace description, that is, with Bragg peaks whose positions can be mapped to a lattice in an arbitrary but finite dimension. Others - eventually the majority - suspected there might be stranger structures out there than those imagined in superspace philosophy, and argued that it would be unwise to replace one inadequate definition with another that might prove inadequate soon. The Commission agreed on a working definition to be used until the picture clarified: a crystal would be any solid with an 'essentially discrete diffraction diagram'. ('Essentially discrete' means sharp Bragg peaks and possibily a weak continuous background.) In short, said the Commission, if it looks like a duck, walks like a duck and quacks like a duck, it is a duck. (I refer here to the original recommendation of the Commission; the minority recommendation described above has at times and places been used instead.)

Intentionally though implicitly, the Commission was posing a challenging research question for crystallographers, mathematicians, chemists, physicists, materials scientists and maybe artists too: what sorts of structures have essentially discrete diffraction diagrams? Or, more abstractly, what characterizes the point sets with this property? The paper by Grimm (2015) is a comprehensive update on this question. Much has been learned in the last quarter century. Indeed, Grimm suspects, even the duck definition may be overly restrictive.

The first task of this research program was to define its terms rigorously even if 'crystal' could not be. 'Order' also eludes precise definition, but in the language in which we speak of it can be made precise. Keywords like 'diffraction', 'Bragg peak' and 'autocorrelation' now have rigorous definitions, which in turn permit rigorous proofs and carefully constructed examples and counter-examples. Grimm gives the reader the background needed to grasp the measure-theoretic definition of diffraction spectrum (diffraction measure), and uses it to frame the rest of the paper. The frame rests on Lebesgue's Decomposition Theorem that a measure is a unique sum of three components, designated pp (pure point - think Bragg peaks), ac (absolutely continuous - think hazy background) and sc (singular continuous - whatever remains in the measure when its pp and ac components are deleted). In this context, Grimm shows us some surprises. None of these has been found in nature yet - the examples are linear sequences generated by substitution rules. But then, Penrose tilings preceded quasicrystals. In the future, who knows?

(1) Point sets with entirely pure point diffraction. The best-known family of pure-point sets is the standard cut-and-project sets, or model sets. Model sets are derived from $n$ dimensional lattices by cutting the lattice with a well-chosen three-dimensional subspace and projecting certain (again well chosen) lattice points onto it. All model sets are crystals under the new definition of crystal: that is, their sc and ac components are empty. Their diffraction measure is thus pure point. But - surprise! - model sets are not the only 


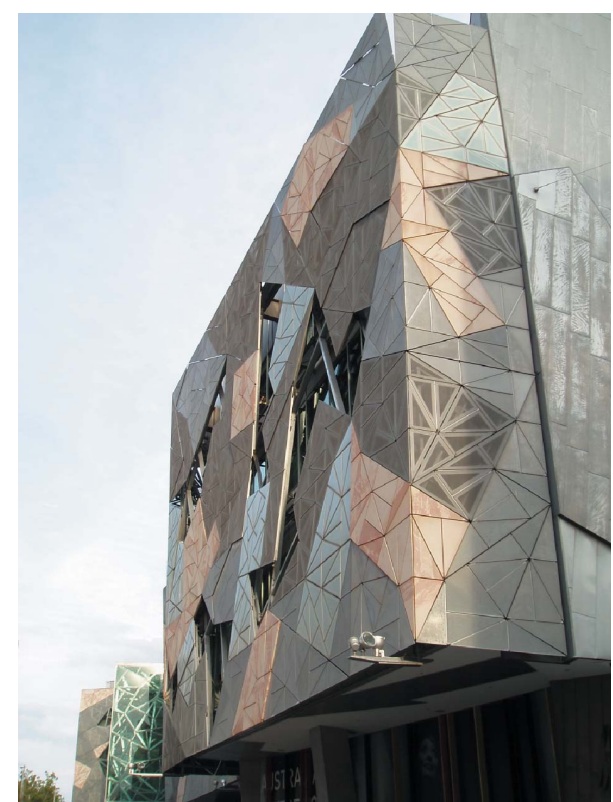

Figure 1

A building at Melbourne's Federation Square featuring a pinwheel tiling façade. To date, there is no complete characterization of the diffraction spectrum of this example. Reproduced with permssion from Grimm (2015).

point sets with the pure-point property. Counterexamples include pure-point sets whose Bragg peaks cannot be located in any finite-dimensional superspace. Among these are the well known 'chair tiling', and linear sequences defined by 'the period doubling substitution rule' (described in detail).

(2) Point sets with singular continuous diffraction. Singular continuous point sets have no Bragg peaks, yet they have distinctive long-range order. Grimm illustrates this with the family of so-called generalized Thue-Morse sequences, again generated by a certain substitution rule. 'Clearly, the gener- alized Thue-Morse systems possess hierarchical order', notes Grimm, 'although this is not reflected in a pure-point component in their diffraction measures. However, this 'hidden' order is visible in other correlations. Explicitly, it can be revealed by looking at the two-point correlations of pairs rather than of single points.'

(3) Point sets with absolutely continuous diffraction. A point set generated by flips of a fair coin has an absolutely continuous diffraction measure, as we expect from a random set. But just as not all point sets with pure point diffraction are model sets, not all point sets with absolutely continous diffraction patterns are stochastic. One of the big surprises (in this field) in recent decades was the discovery that the deterministic Rudin-Shapiro sequence, generated by recursion, and the Bernoulli coin-flipping sequence have the same diffraction diagrams: in older terminology, they are homometric sets.

Research continues (see Fig. 1), but these examples suggest that diffraction (as in the Commission's duck definition) may not be up to the task of characterizing crystal structures. Significantly, although their diffraction spectra are identical, the dynamical spectra of the Rudin-Shapiro and Bernoulli sets are not. For pure-point sets, the two spectra coincide, but for more general structures the dynamical spectrum is richer. The caution of the 1991 Commission on Aperiodic Crystals is, in hindsight, justified but they too did not dream of everything. The next time a Commission tackles this question 'What is a crystal?' it can go deeper.

\section{References}

Grimm, U. (2015). Acta Cryst. B71, 258-274.

Janner, A. \& Janssen, T. (1977). Phys. Rev. B, 15, 643-658.

Shechtman, D., Blech, I., Gratias, D. \& Cahn, J. (1984). Phys. Rev. Lett. 53, 1951-1953.

Wolff, P. M. de (1974). Acta Cryst. A30, 777-785. 